\title{
Techno-Economic Evaluation of Energy Storage Systems Built from EV Batteries - Prospective Revenues in Different Stationary Applications
}

\author{
Tam Thanh Nguyen ${ }^{1,2}$, Maik Naumann, Cong Nam Truong ${ }^{1}$, and Andreas Jossen ${ }^{1}$ \\ ${ }^{1}$ Institute for Electrical Energy Storage Technology at Technical University of Munich (TUM), 80333 Munich, Germany \\ ${ }^{2}$ BMW Group, Petuelring 130, 80788 Munich, Germany
}

\begin{abstract}
Battery energy storage systems (BESSs) are already being deployed for several stationary applications in a technically and economically feasible way. This paper focuses on the revenues of industrial BESSs built from electric vehicle lithium-ion batteries with varying states of health. For this analysis, a stationary BESS simulation model is used, that is parameterised with parameters of a $22-\mathrm{kWh}$ automotive battery. The comprehensive model consists of several detailed sub-models, considering battery characteristics, ageing and operating strategies, which allow technical assessment through time series simulation. Therefore, capacity fade and energy losses are considered in this techno-economic evaluation. Potential economically feasible applications of new and second-life batteries, such as photovoltaic home storage, intraday trading and frequency regulation as well as their combined operation are compared. The investigation includes different electricity price scenarios. The combined operation, followed by frequency regulation, is found to have the highest economic viability for the specified electric vehicle battery.
\end{abstract}

\section{Introduction}

New manufactured and used batteries from electric vehicles (EV), also called second-life batteries, have the potential to become particularly beneficial for the energy transition. Due to increasing production capacities and declining battery costs, new manufactured automotive batteries based on lithium-ion (Li-ion) are expected to be available for stationary usage in battery energy storage systems (BESSs) in the future.

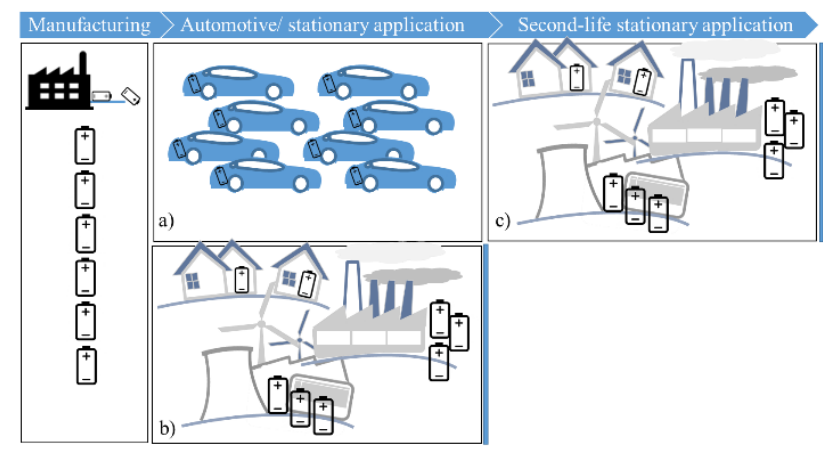

Fig. 1. Automotive Li-ion batteries for several applications: a) first usage in electric vehicle; b) or first usage in BESS performing different stationary applications; c) second usage in several second-life BESS.

In addition, there are retired batteries, which have a decreased capacity through ageing during first use. Instead of recycling them, they were found to be suitable for second use, since they still retain significant capacity. Implemented in a BESS, they are capable to fulfil diverse applications. An overview and description of the most common applications for $\mathrm{Li}$-ion-based BESS is given in [1]. Figure 1 summarises and schematically shows the possible stages of deployment for batteries.

In this regard, synergies between the automotive and power generation sector arise. This paper focuses on the technical evaluation and economic benefit of BESSs built from commercial automotive battery packs of varying states of health ( $\mathrm{SOH})$. The research objective is to com-pare their revenues in several applications in order to determine possible revenues of EV batteries, depending on associated performance impairment and their $\mathrm{SOH}$. The economic analysis is underpinned by technical simulations, which reflect the state of charge (SOC), the $\mathrm{SOH}$ and the storage energy over time. Technical aspects, such as system losses, cooling losses and continuous reduced capacity through ageing depend on the respective operating behaviour and strategy of the respective application and are crucial for the economic analysis. Under consideration of legal regulations and technical framework, the following applications are evaluated: Photovoltaic (PV) home storage, intraday market (IDM) and primary control reserve (PCR) as well as their combined operation (Combined) are compared through a technical and economic analysis.

For this purpose, a techno-economic model, called SimSES, depicting the above applications, is developed. The study includes the evaluation of varying $\mathrm{SOHs}$, 
which is an indication of different dates of removal from the EV, different driving behaviour and different mileages.

At present, there are a number of papers, which review energy storage technologies and summarise options for stationary applications. Similar model approaches in literature often solely discuss PV systems and partially neglect Li-ion ageing [3, 4]. Therefore, this research work will highlight handled cumulated energy, energy losses and degradation after a lifetime of twenty years. The simulations model the full complexity of the BESS's operational behaviour and consider technical and economic levers of four different applications.

The contribution is organised as follows. In Section 2, a brief overview about the techno-economic model and its implemented functions is given. In Section 3, technical input parameters of simulated stationary applications are presented. Section 4 displays the simulation results. Finally, Section 5 gives the conclusions to the analysis.

\section{Simulation model}

The utilised BESS simulation model SimSES [2] is structured in several sub-models: equivalent circuit battery model, empirical ageing model depicting calendar and cycle ageing, power electronics model and thermal model for the whole system. The sub-models are coupled in order to reflect the high interdependencies and to describe the system behaviour as accurately as possible. Figure 2 describes the simulation framework with all sub-models. Further information about the utilised power flow simulation model can be found in [2] and [5].

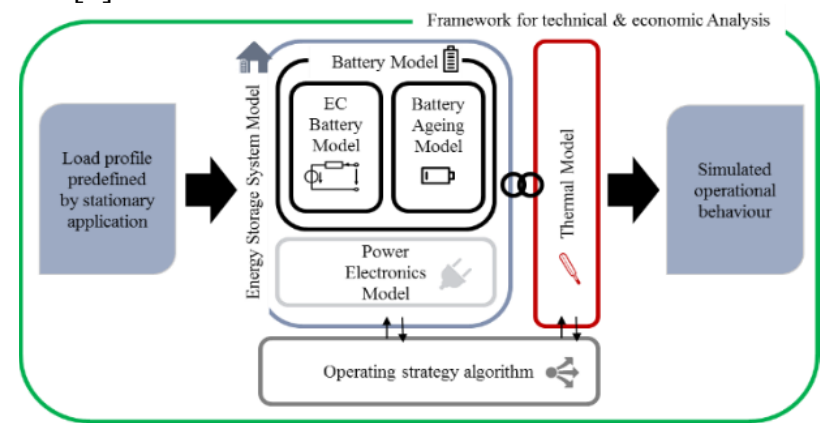

Fig. 2 Simulation framework for technical and economic analysis.

\section{Technical model input of stationary applications}

The simulation model is parameterised with typical performance characteristics of an automotive $22-\mathrm{kWh}$ Li-ion battery pack from Table 1 . The power flow is calculated and saved for each use case. The capacity loss is observed for a simulated operation of twenty years, knowing well, that the remaining lifetime of the reused Li-ion storage might not be sufficient with the little remaining $\mathrm{SOH}$. It shall be investigated, which applications are more stressful to the battery pack than others and if a common End of Life (EoL) limitation at eighty percent remaining useful life is practical or not.
The model input for each of the simulated stationary applications: PV home storage, IDM, and PCR are briefly summarised in Table 2-4.

Precise simulations in an hourly time series are done with the parameter set from Table 2 matching a typical building with 4-6 rental parties with an $8-\mathrm{kWp}$ rooftop $\mathrm{PV}$ installation. PV-generated surplus energy is charged and afterwards discharged, as soon as the household load exceeds the generated power by the PV system. Due to the highly volatile pricing in the home storage area, the economic model is executed with four electricity price scenarios for the residential use case. Thus, several possible price ranges for electricity price and feed-in rates in the future are evaluated.

An IDM strategy based on electricity prices from 2017 is implemented as a recharging strategy for scenarios where the storage needs to fulfil a load requirement in order to guarantee German primary control without any impairments. The standalone IDM application was also simulated, but as the revenues did not show to be profitable in Germany except with very precise electricity price forecasts, the use case is not further elaborated. The profitability might be different in other countries like in the U.S. where electricity prices commonly change fivefold depending on time, season and service territory.

The German PCR application is simulated in a quarterhourly time series unlike the others due to the billing interval which is every 15 minutes.

The last investigated application constitutes a combined operation of all previous three applications. The PCR application has priority because of high remuneration and penalty reasons. PV home storage and IDM follow in this order as recharging strategies to meet the SOC band around 55\% SOC for PCR. The same model input according to Table 1-4 is taken as the basis. Table 5 depicts the application hierarchy. The energy and cash flow calculations are done for a medium-sized standard battery pack.

Table 1. Model input: battery pack.

\begin{tabular}{|l|l|}
\hline Technology & Li-ion \\
\hline Nom. energy capacity & $22 \mathrm{kWh}$ (partially pooling) \\
\hline Start SOH & $50 \%-100 \%$ \\
\hline Lifespan simulated & $20 \mathrm{a}$ \\
\hline
\end{tabular}

Table 2. Model input: PV home storage.

\begin{tabular}{|l|l|}
\hline $\begin{array}{l}\text { Installed PV peak } \\
\text { power }\end{array}$ & $8 \mathrm{kWp}$ (common size [6]) \\
\hline Annual Load & $7 \mathrm{MWh}$ (common size [6]) \\
\hline $\begin{array}{l}\text { Electricity price } \\
\text { scenarios }\end{array}$ & $\begin{array}{l}\text { Constant increase/ linear } \\
\text { extrapolation of historical el. } \\
\text { price }\end{array}$ \\
\hline Feed-in rate & $\begin{array}{l}12,20 \mathrm{ct} / \mathrm{kWh}(\mathrm{Q} 1 \text { 2018) } \\
3 \mathrm{ct} / \mathrm{kWh}(\mathrm{el} . \text { market price) }\end{array}$ \\
\hline
\end{tabular}

Table 3. Model input: intraday market (IDM).

\begin{tabular}{|l|l}
\hline IDM electricity price & based on prices from 2017
\end{tabular} 
Table 4. Model input: German primary control (PCR).

\begin{tabular}{|l|l|}
\hline Power-to-energy ratio & $1: 2.5$ \\
\hline Frequency response & $\begin{array}{l}\text { based on frequency regulation } \\
\text { from 2015 (repetitive) }\end{array}$ \\
\hline PCR compensation & $\begin{array}{l}\text { Based on rate from 2017 } \\
\text { - minimum price scenario } \\
\text { - maximum price scenario } \\
\text { - winning bid every week }\end{array}$ \\
\hline
\end{tabular}

Table 5. Model input: combined operation (Combined).

\begin{tabular}{|l|ll|}
\hline Application hierarchy & 1. & primary control reserve \\
(implemented in control & 2. PV home storage \\
strategy) & 3. intraday market \\
\hline
\end{tabular}

Table 6. Technical results: photovoltaic (PV) home storage.

\begin{tabular}{|c|c|c|c|c|c|c|c|}
\hline 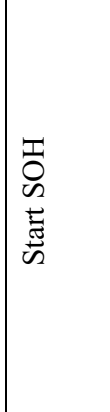 & 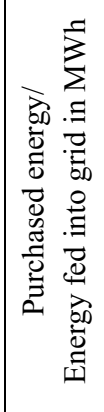 & 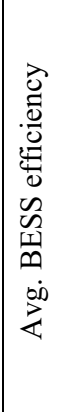 & 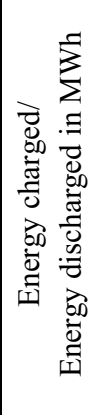 & 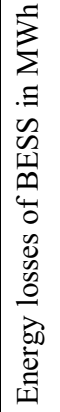 &  & 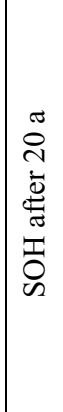 & 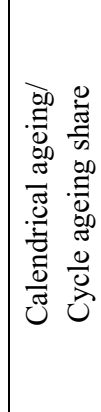 \\
\hline $100 \%$ & 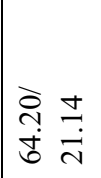 & $\begin{array}{l}\stackrel{0}{\partial} \\
\text { ָे } \\
\infty\end{array}$ & 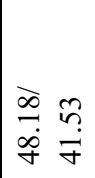 & $\begin{array}{l}\overrightarrow{0} \\
\dot{0}\end{array}$ & $\begin{array}{l}\vec{n} \\
\text { di }\end{array}$ & $\begin{array}{l}* \\
0 \\
0 \\
\infty \\
0 \\
i \\
i n\end{array}$ &  \\
\hline $90 \%$ & 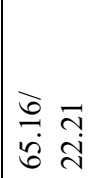 & $\begin{array}{l}\stackrel{0}{\grave{ \pm}} \\
\dot{0} \\
\infty\end{array}$ &  & ñn & $\begin{array}{l}\vec{\infty} \\
\vec{d}\end{array}$ & $\begin{array}{l}* \\
0 \\
8 \\
\dot{8} \\
i n\end{array}$ & $\bar{n} \stackrel{a}{f}$ \\
\hline $80 \%$ & 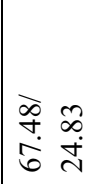 & $\begin{array}{l}0 \\
0 \\
8 \\
0 \\
\infty\end{array}$ & 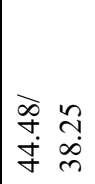 & $\hat{\imath}$ & $\begin{array}{l}0 \\
\text { aे } \\
\text { in }\end{array}$ & 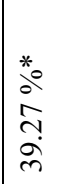 & $\begin{array}{l}\infty \\
+\infty \\
+ \\
0\end{array}$ \\
\hline $70 \%$ & 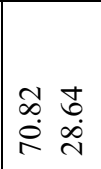 & $\begin{array}{l}0^{0} \\
n \\
\infty \\
\infty \\
\infty\end{array}$ & 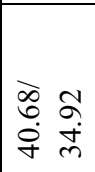 & $\frac{n}{i n}$ & $\mid \begin{array}{l}\infty \\
\infty \\
\infty \\
-1\end{array}$ & 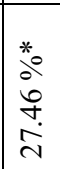 & $\begin{array}{l}\text { J } \\
0 \\
0\end{array}$ \\
\hline $60 \%$ & $\begin{array}{ll}\vec{f} & 0 \\
i & \infty \\
i & m\end{array}$ & $\begin{array}{l}00 \\
m \\
\ddot{\infty} \\
\infty\end{array}$ & 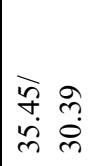 & $\begin{array}{l}0 \\
0 \\
\dot{n}\end{array}$ & $\underset{f}{\vec{f}}$ & $\begin{array}{l}* \\
0 \\
0 \\
6 \\
i \\
n\end{array}$ & $\begin{array}{ll}\bar{\sigma} & \overline{0} \\
\tilde{0} & 0\end{array}$ \\
\hline $50 \%$ & $\begin{array}{ll}\stackrel{\partial}{+} & \infty \\
\dot{\infty} & \stackrel{+}{+}\end{array}$ & $\begin{array}{l}00 \\
0 \\
0 \\
n \\
\infty \\
\infty\end{array}$ & 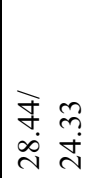 & $\exists$ & $\stackrel{n}{n}$ & 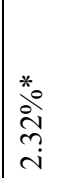 & $\begin{array}{l}\hat{m} \\
\hat{0} \\
0\end{array}$ \\
\hline 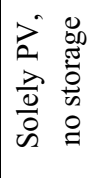 & 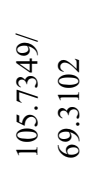 & & & & & & \\
\hline
\end{tabular}

\section{Results}

The results of the conducted simulations and the economic potential derived from those are presented for each of the applications in the following.

Table 6, 7 and 8 comprise cumulated power flows of purchased energy and fed-in grid energy from the consumer viewpoint as well as charged energy, discharged energy and energy losses from the energy storage viewpoint in MWh after 20 years. Furthermore, average efficiency and number of equivalent full cycles (EFC), calculated

Table 7. Technical results: German PCR.

\begin{tabular}{|c|c|c|c|c|c|c|c|}
\hline 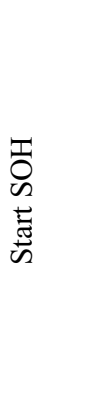 & 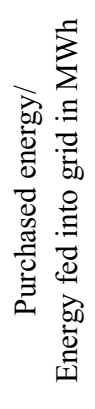 &  & 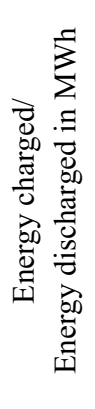 & 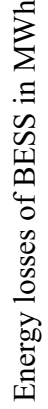 & 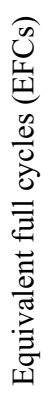 &  & 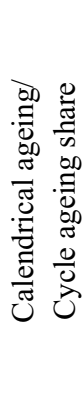 \\
\hline $100 \%$ & $\stackrel{\bar{m}}{\overrightarrow{0}}$ & $\begin{array}{l}\stackrel{\circ}{\grave{a}} \\
\text { ì }\end{array}$ & 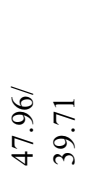 & $\stackrel{\sim}{\infty}$ & $\begin{array}{l}\text { ָे } \\
\text { i }\end{array}$ & 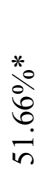 & $\begin{array}{c}\text { के } \\
0 \\
0\end{array}$ \\
\hline $90 \%$ & $\stackrel{\bar{m}}{\overrightarrow{0}}$ & $\begin{array}{l}\text { ìं } \\
i n \\
i \\
\infty\end{array}$ & 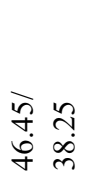 & $\stackrel{\text { ஸे }}{\infty}$ & $\frac{i}{i}$ & $\begin{array}{l}* 0 \\
\stackrel{0}{0} \\
\dot{\infty} \\
\dot{J}\end{array}$ & $\begin{array}{c}\tilde{\infty} \\
0 \\
0\end{array}$ \\
\hline $80 \%$ & $\stackrel{\bar{\imath}}{\overrightarrow{0}}$ & $\begin{array}{l}\text { iें } \\
\text { aे }\end{array}$ & 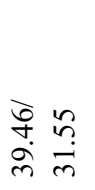 & $\overline{\widehat{\sigma}}$ & ֻ & $\begin{array}{l}\frac{*}{0} \\
\stackrel{\circ}{a} \\
\dot{0} \\
0\end{array}$ & $\stackrel{\infty}{\infty}=$ \\
\hline $70 \%$ & $\exists \overline{0}$ & 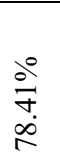 & 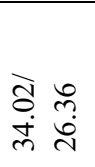 & $\begin{array}{l}0 \\
\stackrel{0}{0}\end{array}$ & $\stackrel{+}{n}$ & 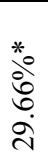 & $\begin{array}{ll}\text { ते } & \infty \\
\stackrel{0}{0} & 0 \\
\end{array}$ \\
\hline $60 \%$ & $\begin{array}{l}\exists \\
0\end{array}$ & $\frac{0^{\circ}}{n}$ & 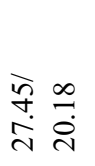 & $\stackrel{ָ}{N}$ & 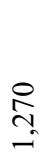 & $\begin{array}{l}\stackrel{*}{0} \\
\dot{D} \\
\infty \\
\dot{\lambda} \\
\dot{\lambda}\end{array}$ & $\stackrel{\infty}{\infty} \stackrel{0}{0}$ \\
\hline $50 \%$ & $\begin{array}{ll}0 & 0 \\
0 & 0 \\
0 & 0\end{array}$ & 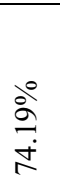 &  & $\frac{12}{6}$ & $\infty$ & $\begin{array}{l}\dot{0} \\
\dot{0} \\
\text { in }\end{array}$ & $\stackrel{ }{\hat{*}} \stackrel{\overbrace{}}{0}$ \\
\hline
\end{tabular}

$$
\eta=1-E_{\text {losses }} / E_{\text {in-stored }}
$$

"Limitation: Ageing model is not validated for lower SOH. It is anticipated, that the current rates and operational voltage windows during stationary operation are much smaller than that during use in the vehicle, thus implying a linear character of the cell ageing behaviour under standard conditions. 


$$
N_{e q}=W_{t o t} /\left(2 U_{N} \cdot C_{N}\right)
$$

are presented in order to evaluate the BESS performance.

The remaining degradation parameters indicate the remaining $\mathrm{SOH}$ after 20 years and the ratio of calendar and cycle ageing. Due to the fact that electric vehicles have not been on the market for more than four to five years, the ageing model could not be validated for $\mathrm{SOH}$ far below $60 \%$. Still, there are ageing studies of single cells, which indicate, that an optimal operation in stationary BESSs may avoid or retard nonlinear aging characteristics near approximately $80 \% \mathrm{SOH}$ and extend the life of Li-ion cells [7]

Table 8. Technical results: combined operation (combined).

\begin{tabular}{|c|c|c|c|c|c|c|c|}
\hline $\begin{array}{l}\text { D } \\
\text { 足 } \\
\text { 志 } \\
\text { in }\end{array}$ & 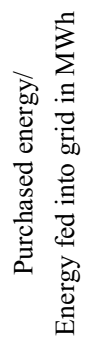 & 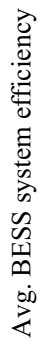 & 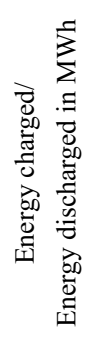 & 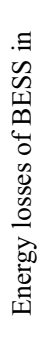 &  & 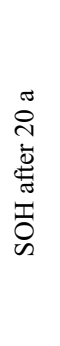 & 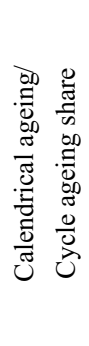 \\
\hline $100 \%$ & 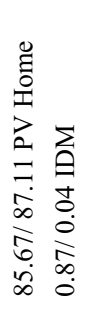 & 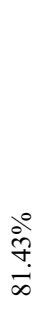 & 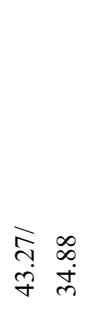 & $\overbrace{\infty}$ & $\begin{array}{l}\text { ô } \\
\text { i }\end{array}$ & 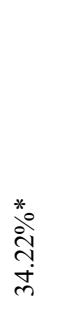 & $\begin{array}{ll} & = \\
\infty & = \\
0 & 0\end{array}$ \\
\hline $90 \%$ & 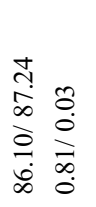 & $\begin{array}{l}\stackrel{0}{\circ} \\
\dot{\infty}\end{array}$ & $\begin{array}{ll}\vec{\infty} & \overrightarrow{\vec{n}} \\
\dot{\sigma} & \vec{m}\end{array}$ & $\underset{m}{n}$ & $\stackrel{\infty}{\infty}$ & 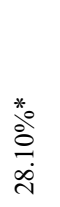 & 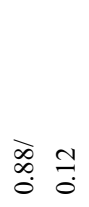 \\
\hline $80 \%$ & 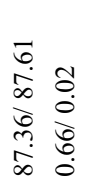 & ळें &  & $\begin{array}{c}\pi \\
\infty \\
\infty\end{array}$ & 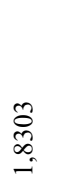 & $\begin{array}{l}\stackrel{*}{\circ} \\
\stackrel{0}{\Xi} \\
\stackrel{-}{=}\end{array}$ & $\begin{array}{ll}\text { ते } \\
\infty \\
0 & 0 \\
0 & 0\end{array}$ \\
\hline $70 \%$ & 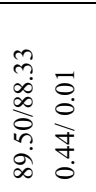 & $\begin{array}{c}\text { ळें } \\
\text { ळे } \\
\infty\end{array}$ &  & $\stackrel{\text { aे }}{\therefore}$ & 弈 & $\begin{array}{l}\frac{*}{\circ} \\
\stackrel{0}{\circ} \\
\stackrel{0}{0}\end{array}$ & $\begin{array}{ll}\text { Fo } & 0 \\
0 & 0 \\
0 & 0\end{array}$ \\
\hline $60 \%$ & 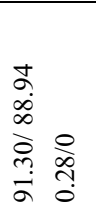 & $\stackrel{\stackrel{\circ}{N}}{\stackrel{r}{r}}$ & 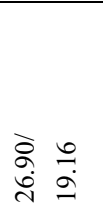 & 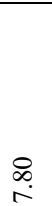 & $\stackrel{\substack{m \\
m}}{m}$ & $\begin{array}{l}\stackrel{0}{\circ} \\
\stackrel{\circ}{N} \\
\stackrel{0}{0}\end{array}$ & $\begin{array}{ll}\text { for } \\
0 \\
0\end{array}$ \\
\hline
\end{tabular}
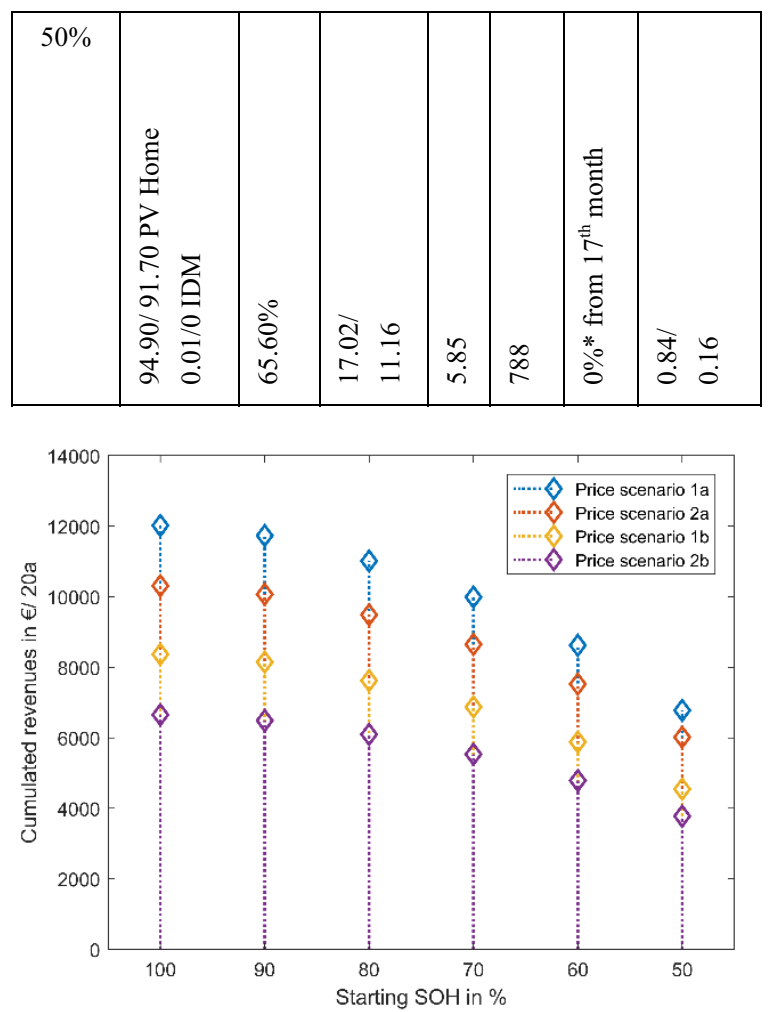

Fig. 3. Cumulated PV home storage revenues of a $22-\mathrm{kWh}$ EV battery after a 20 -year lifespan under several price scenarios:

1) linear extrapolation of historical electricity prices;

2) constant increase of historical electricity prices;

a) feed-in remuneration Q1 from 2018;

b) electricity market feed-in remuneration

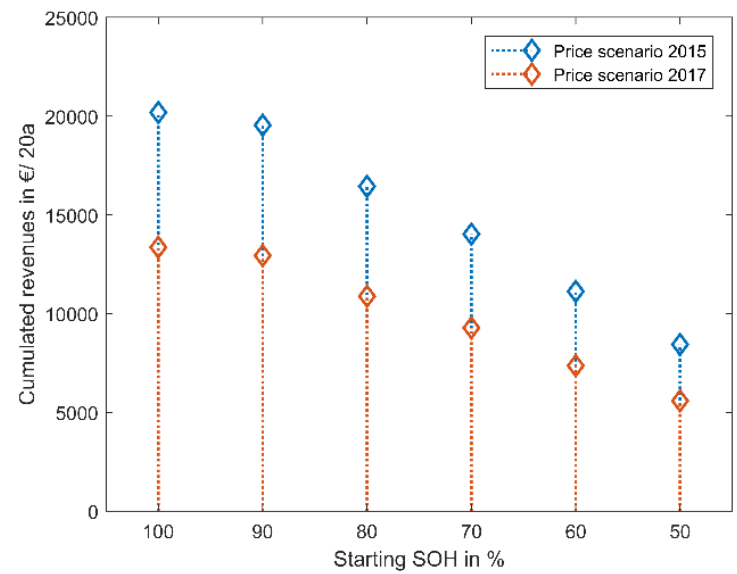

Fig. 4. Cumulated German primary control revenues of a 22$\mathrm{kWh}$ EV battery after a 20 -year lifespan for the historic price scenarios from 2015 and 2017. 


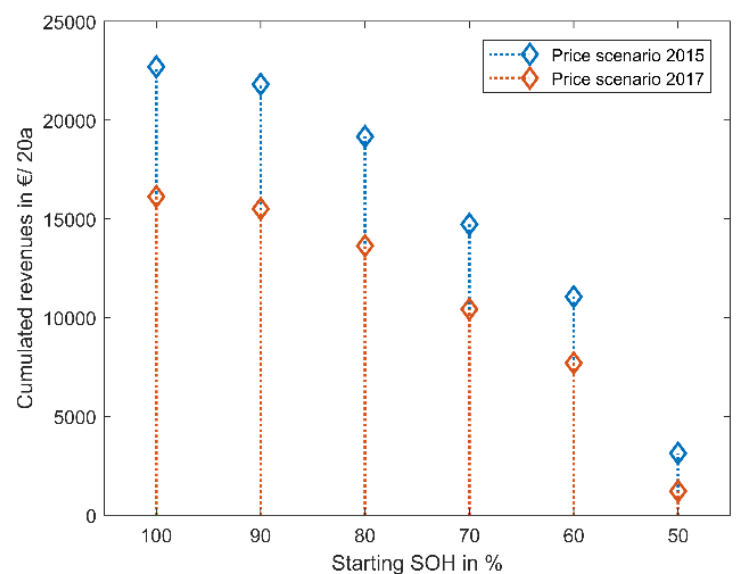

Fig. 5. Cumulated combined operation revenues of a $22-\mathrm{kWh}$ EV battery after a 20 -year lifespan for the historic price scenarios from 2015 and 2017; PV home storage price scenario 1a) from Fig. 3 is taken as presumption.

The difference in energy between the last line of Table 6 to the others in the same column depicts the improvement in self-consumption compared to a PV system without energy storage. Using an energy storage system requires 39\% less energy purchases and 69\% less energy exports from PV generation to the grid compared to solely PV.

The self-sufficiency and cumulated energy decreases with lower initial SOH. Naturally, there are the most number of EFCs and the highest system efficiencies for battery packs with more remaining capacity.

The capacity loss is the highest for the combined operation indicating the most stressful application for the storage due to the greatest utilisation rate overall. In terms of accelerated ageing, the combined operation is followed by the PV home storage application. The applications show a higher capacity loss of $39-48 \%$ and more than 59\%, respectively. The PV home storage application with characteristics of high power demand and, therefore, a higher share of cycle ageing seem to have a higher impact on lifetime than the PCR application with a continuous and less demanding load profile.

Whether the more aggressively cycling works out to be more economical because more revenue is earned, is better comprehensibly when translating the cumulated energy and ageing behaviour into revenues by means of the economic model. A depreciation period of 20 years is taken because this is the standard service life of PV systems [8]. Further, interest and inflation rates are estimated as mentioned in Table 9 according to other publications [9-12]. The cumulated revenues are calculated as follows:

$\operatorname{sum}($ revenues $)=\Sigma$ t cash flow $(t) \cdot(1+\text { interest rate })^{-t}$

with $t$ representing the number of elapsed years.

Table 9. Economic model input.

\begin{tabular}{|l|l|}
\hline Interest rate & $4 \%$ p.a. \\
\hline Inflation rate & $2 \%$ p.a. \\
\hline Depreciation period & 20 years \\
\hline
\end{tabular}

Figure 3, 4 and 5 present the cumulated PV home storage revenues in Euros $(€)$ of a $22-\mathrm{kWh}$ EV battery after a 20-year lifespan of uninterrupted operation in order to estimate the maximum allowable battery and installation costs for each of the applications. Different economic scenarios are considered in terms of electricity prices. Looking at the results, the combined operation is found to be the application with the highest economic viability followed by German PCR. This is in good agreement with the recent results from Shi et al. [13]. Further, taking also the operating strategy and capacity loss due to ageing into account, PCR represents the sturdier application with high profits and lesser stress on the BESS implying lower costs for maintenance or replacement of faulty battery packs. Another remarkable finding is that the difference in profit between $100 \%$ and $80 \%$ initial $\mathrm{SOH}$ accounts to less than $8.36-8.40 \%$, $15.46-15.52 \%$ and $18.50-18.51 \%$ for PV home storage, combined and German PCR operations, respectively. This result reaffirms the suggested EoL found in literature at below $80 \% \mathrm{SOH}$, especially for the PV home storage and combined applications. The simulations with $50 \%$ initial $\mathrm{SOH}$ show much lower profits than the other simulations in addition to not being durable enough for a 20 -year lifetime. It is advised to replace those battery packs during second use upon exhaustion in order to receive higher returns for operation.

\section{Conclusion}

This paper concludes that the combined application is the most economically feasible for the deployment of EV battery packs.

Contrasting to the above, the PV home storage use case only shows a positive return on invest if the overall system costs (incl. installation and maintenance) do not exceed 6,000-11,000€ for an $80 \%$ initial $\mathrm{SOH}$.

When accounting for battery ageing, the German PCR application is most beneficial based on the conducted techno-economic simulations. A net present value (NPV) of $10,000-18,000 €$ per battery pack is estimated on the basis of an $80 \%$ initial SOH. The preceding German PCR time series simulations reveal a plausible 20-year lifespan for a $50-100 \%$ initial $\mathrm{SOH}$. This conclusion can be made at this stage, but project planning for future BESSs needs to consider that the German PCR market is limited.

By means of the comprehensive embedded ageing model, the suggested, but in literature still unverified EoL of $80 \% \mathrm{SOH}$, can be affirmed. Further, other estimated NPVs of a new or used EV battery pack are given for all PV home storage, IDM, PCR and combined variations in $\mathrm{SOH}$ and aforementioned pricing scenarios. The technical simulations remain limited for the prediction of nonlinear ageing, single cell failures and unforeseeable failures due to other individual components. 


\section{References}

1. Bauer, M. and Nguyen, T.T., Jossen, A., Lygeros, J.: Evaluating frequency regulation operated on two stationary energy storage systems with batteries from electric vehicles, Journ. of Energy Procedia (to be published)

2. M. Naumann, C. N. Truong, M. Schimpe, D. Kucevic, A. Jossen and H. C. Hesse. SimSES: Software for techno-economic Simulation of Stationary Energy Storage Systems. (International ETG Congress, Bonn, 2017)

3. Madlener, R., Kirmas, A.: Economic viability of second use electric vehicle batteries for energy storage in residential applications. Journ. of Energy Procedia 105, 3806 - 3815 (2017)

4. Hoppmann, J., Volland, J., Schmidt, T.S., Hoffmann, V.H.: The economic viability of battery storage for residential solar photovoltaic systems - A review and a simulation model. Journ. of Renewable and Sustainable Energy Reviews 39, 1101-1118 (2014)

5. Truong, C.N., Naumann, M., Karl, R.Ch., Müller, M., Jossen, A., Hesse, H.C.: Economics of Residential Photovoltatic Battery Systems in Germany: the Case of Tesla's Powerwall. Batteries 2, 14 (2016)

6. Energie-Info: Stromverbrauch im Haushalt; (Bundesverband der Energie- und Wasserwirtschaft e.V.: Berlin, in German, 2014)
7. S.F. Schuster, T. Bach, E. Fleder, J. Müller, M. Brand, G. Sextl, A. Jossen, Nonlinear aging characteristics of lithium-ion cells under different operational conditions, Journ. of Energy Storage 1, 44-53 (2015)

8. Bundesministerium der Finanzen. (AfA-Tabelle für die Allgemein Verwendbaren Anlagegüter ("AV"); Juris: Saarbrücken, in German, 2000)

9. Diaf, S.; Belhamel, M.; Haddadi, M.; Louche, A. Technical and economic assessment of hybrid photovoltaic/wind system with battery storage in Corsica island. Energy Policy 36, 743-754 (2008)

10. Dufo-López, R. Optimisation of size and control of grid-connected storage under real time electricity pricing conditions. Appl. Energy 140, 395-408 (2015)

11. Yang, H.; Wei, Z.; Lou, C. Optimal design and techno-economic analysis of a hybrid solar-wind power generation system. Appl. Energy 86, 163-169 (2009)

12. Zucker, A.; Hinchliffe, T. Optimum sizing of PVattached electricity storage according to power market signals - A case study for Germany and Italy. Appl. Energy 127, 141-155 (2014)

13. Shi, Y., Xu, B., Wang, D., Zhang, B.: Using Battery Storage for Peak Shaving and Frequency Regulation: Joint Optimization for Superlinear Gains, IEEE Trans. on Power Systems 33 (2017). 